\title{
Heavy flavours and QCD studies at HERA
}

\author{
Volodymyr Aushev ${ }^{1}$ \\ DESY/KINR \\ Notkestrasse 85, D-22607 Hamburg, Germany \\ E-mail: aushev@mail.desy.de
}

In deep-inelastic scattering of electrons (DIS) off protons at the HERA collider the production of heavy flavours plays an important role and it is well suited to study the properties of the theory of QCD. At HERA energies, these flavours include in particular the charm quarks and even heavier beauty quarks. Precise knowledge of the proton structure is of utmost importance for the interpretation of LHC data. The proton parton density functions (PDFs) are determined at HERA with high accuracy using the method of deep-inelastic scattering. Charm quarks contribute up to $30 \%$ to the total DIS cross section through their production in the bosongluon fusion process, in which gluons from the proton are directly involved. Therefore charm quark physics is very important for the QCD analysis of the proton structure, which is intricate due to theoretical ambiguities in the treatment of heavy quarks. The production of beauty is measured too, and the beauty contribution to the proton structure function is calculated. Measurements of differential cross sections for jet production have allowed detailed studies of parton dynamics, tests of the proton and photon parton distribution functions. This also provides additional precision information for the determination of the strong coupling constant and the measurement of the proton structure.

The XXth International Workshop High Energy Physics and Quantum Field Theory Sochi, Russia

September 24-October 1, 2011

\footnotetext{
1 Speaker, on behalf of the ZEUS collaboration
} 


\section{Introduction}

For 15 years, electrons and protons collided at extremely high energies inside the particle accelerator ring HERA. Research operation was concluded in summer 2007 and HERA was switched off. The evaluation of the recorded data, however, is continuing at full speed. During that time, many of the insights provided by HERA into the microcosm found their way into the physics textbooks. They are now part of the basic knowledge we have of the workings of our world. The HERA physicists are now perfecting a comprehensive overall picture of the proton and the forces acting within it - with a precision that won't be matched by any other particle accelerator in the world for years to come.

\subsection{The ZEUS detector at HERA}

\subsubsection{The HERA Collider}

The HERA (Hadron-Elektron Ring-Anlage) storage ring facility at DESY was the only one in the world in which two different types of particles were accelerated separately and then brought to collision. It was built at the DESY (Deutsches Elektron Synchrotron) laboratory in Hamburg, Germany between 1984 and 1991, and was in operation from 1992 to 2007. Here, in a 6.3-kilometer-long tunnel, lightweight electrons (or their antiparticles, the positrons), accelerated to an energy of $27.5 \mathrm{GeV}$, collided with $820(920) \mathrm{GeV}$ protons. In these electronproton collisions, the point-like electron acts like a tiny probe that scans the inside of the proton and reveals its inner structure. The resulting centre-of-mass energy was 300(318) GeV, more than an order of magnitude higher than the previous fixed-target experiments, which allowed a wider kinematic range in $x$ and $Q^{2}$ to be explored. The higher the energy of the particle collision, the deeper physicists are able to gaze into the proton, and the more details are revealed. That's why HERA was dubbed a "super electron microscope". Thanks to HERA's highly precise "electron probes", particle physicists are able to investigate the inner structure of the proton and the fundamental forces of nature in great detail.

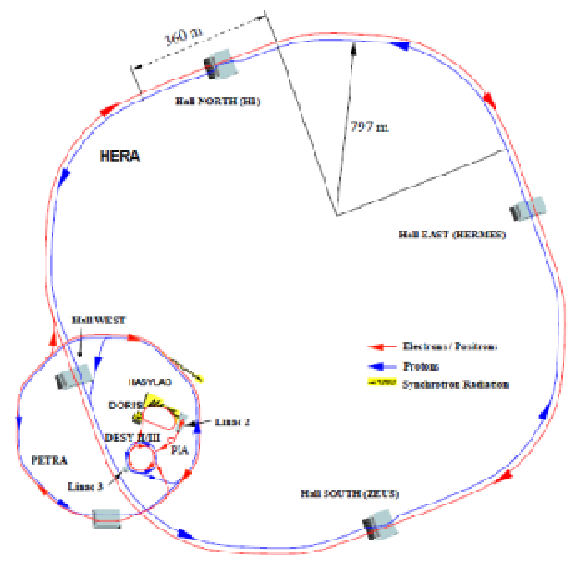

Fig. 1. Schematic view of the HERA collider and its pre-accelerator system.

In 1992, the first two HERA experiments went into operation: $\mathrm{H} 1$ in the North Hall and ZEUS in the South Hall. Both experiments observed the high-energy collisions of electrons and protons in order to unravel the internal structure of the proton and the mysteries of nature's fundamental forces. These detectors were in operation until mid-2007, and between them they recorded a gigantic amount of data. Active data taking has been completed, but the HERA experiments are continuing. Figure 1 shows a schematic drawing of the HERA collider and its pre-accelerator systems. 
The HERA collider had two different injection systems for the beams. The proton acceleration chain started with negative hydrogen ions $\left(\mathrm{H}^{-}\right)$accelerated in a LINAC to an energy of $50 \mathrm{MeV}$. The electrons were then stripped off the $\mathrm{H}^{-}$ions to obtain protons which were injected into the proton synchrotron DESY III and accelerated up to $7.5 \mathrm{GeV}$ in 11 bunches with a temporal gap of $96 \mathrm{~ns}$, the same as in the main HERA ring; these bunches were then transferred to PETRA, where they were accelerated to $40 \mathrm{GeV}$. Finally they were injected into the HERA proton storage ring and the injection stopped when the ring was filled with 210 bunches. In the HERA ring the proton beam was then accelerated up to $920 \mathrm{GeV}$. The leptons were first accelerated to an energy of $250 \mathrm{MeV}$ (electrons) and $450 \mathrm{MeV}$ (positrons) in the linear accelerators LINAC I and LINAC II, respectively. They were then injected to the DESY synchrotron, accelerated to $7.5 \mathrm{GeV}$ and then transferred to PETRA II, where they reached an energy of $14 \mathrm{GeV}$ in bunches separated by $96 \mathrm{~ns}$ gaps. Finally they were injected into the HERA ring, until it contained 210 bunches and accelerated to the nominal lepton beam energy of $27.5 \mathrm{GeV}$. In order to study the background conditions, some of the bunches were kept empty, they are known as pilot bunches. When both bunches were empty the non-beam related background, such as cosmic rays, could be studied. When either the lepton or the proton bunch was empty, it was used to study the beam-related background originating from the interaction of the lepton or proton beam with the residual gas in the beam pipe.

HERA had two phases of operation, known as HERA I and HERA II. The first phase of operation, HERA I, lasted from 1992 to 2000 and the total delivered luminosity during that period was $193 \mathrm{pb}^{-1}$. During the shutdown in 2000/2001, the HERA collider was upgraded to deliver about a factor five times higher specific luminosity at the interaction point. In addition spin rotators were included to rotate the spin of the leptons such that the lepton beam was longitudinally polarized at all interaction regions. The experiments also took the opportunity of the shutdown to upgrade their detectors to achieve higher precision measurements, especially in the context of heavy flavour and high $Q^{2}$ physics. After the detector and accelerator upgrades, the second phase of data taking, HERA II, began in 2003. From October 2003, until the end of 2006, HERA provided stable beam operations and delivered a total luminosity of $556 \mathrm{pb}^{-1}$. Before the end of data taking (June 2007), special data, using two different lower proton beam energies $460 \mathrm{GeV}$ (Low Energy Run) and $575 \mathrm{GeV}$ (Medium Energy Run) were taken. The corresponding luminosities for the LER and MER were $15.8 \mathrm{pb}^{-1}$ and $8.1 \mathrm{pb}^{-1}$, respectively. This data was taken in order to measure the longitudinal structure function, $F_{L}$, of the proton.

\subsubsection{ZEUS Detector}

The ZEUS detector was a multi-purpose detector designed to study a wide range of physics involving high energy lepton-proton collisions at HERA. It had a size of $12 \times 11 \times 20 \mathrm{~m}^{3}$ and a weight of 3600 tons. It was a quasi-hermetic detector as it covered most of the $4 \pi$ solid angle with the exception of very small regions around the beam pipe. As a result of the energy difference between the electron and proton beams, most of the final state particles were boosted in the proton beam direction. Thus the ZEUS detector was specially instrumented with additional sub-detectors in the proton beam direction. The ZEUS coordinate system is a righthanded Cartesian system with the origin at the nominal interaction point. The Z-axis points in the direction of the proton beam and is often referred to as the "forward direction". The X-axis is perpendicular to the beam direction pointing towards the centre of the HERA ring and the Y axis points upwards. The polar angle, $\theta$, and the azimuthal angle, $\varphi$, are measured relative to the $\mathrm{Z}$-axis and $\mathrm{X}$-axis, respectively. In general, the pseudorapidity, $\eta=-\ln \tan (\theta / 2)$, is used instead of the angle $\theta$, as a difference in $\eta$ is longitudinally Lorentz-invariant.

The ZEUS detector had the typical design of high energy physics experiments.

Starting from the interaction point and moving radially outwards, the ZEUS detector consisted of charged particle tracking detectors surrounding the beam pipe. The innermost detector was the vertex detector which was removed during the 1995-1996 shutdown and, in 2001, a silicon 
microvertex detector (MVD) was installed in its place to improve the tracking and especially vertexing. The MVD was surrounded by the central tracking detector (CTD) which was a cylindrical drift chamber. The CTD was supplemented in the forward region by three sets of planar drift chambers (FTD) with interleaved transition radiation detectors (TRD) and in the rear direction with one planar drift chamber consisting of three layers (RTD). In order to improve the forward tracking, in 2001, the TRD system was replaced by a straw-tube. All the tracking components combined, offered an angular acceptance of $7.5^{\circ}<\theta<170^{\circ}$ for charged particles. The tracking system was enclosed by a superconducting solenoid providing a magnetic field of $1.43 \mathrm{~T}$ for the determination of charge and momentum of the charged particles.

A high resolution compensating uranium-scintillator calorimeter (CAL) surrounded the tracking detectors and the magnet. It was the main instrument to measure the energy of electrons and hadrons and was subdivided into three sections, the forward (FCAL), the barrel (BCAL) and the rear (RCAL) calorimeters. Inside of the FCAL and RCAL the hadron-electron separator (HES) was placed, which helped to distinguish electrons from hadrons. In front of the CAL, scintillating tiles, called the presampler were used for the detection of pre-showering particles improving the energy measurement. Further, the CAL was surrounded by the iron yoke which provided the return path for the magnetic field flux and was instrumented with proportional tubes serving as a calorimeter (backing calorimeter, $(\mathrm{BAC})$ ) for the particles which had not been observed by the CAL. The BAC was also used as a muon detection system. Dedicated muon identification chambers were located inside and outside the iron yoke.

The silicon microvertex detector (MVD) was installed in the ZEUS experiment in the gap between the beam pipe and the inner volume of the CTD. The MVD was designed to improve the overall precision of the tracking system and to allow the identification of events containing secondary vertices originating from the decay of particles with long lifetime $(c \tau>$ $100 \mu \mathrm{m})$.

Some of the design specifications of the MVD were:

○ polar angular coverage of $10^{\circ}-160^{\circ}$;

○ measurement of three points for each track in two independent projections;

○ $20 \mu \mathrm{m}$ intrinsic hit resolution;

○ two-track separation of $200 \mu \mathrm{m}$.

The barrel MVD was equipped with 600 single-sided silicon strip sensors of approximately $64 \times 64 \mathrm{~mm}^{2}$ size and $320 \mu \mathrm{m}$ thickness with $\mathrm{p}^{+}$strips implanted into n-type bulk. Each sensor had 3082 strips with $20 \mu \mathrm{m}$ pitch. Every sixth strip was read out resulting in 512 readout strips at a readout pitch of $120 \mu \mathrm{m}$. The MVD silicon sensors were read out using the analog chips Helix 3.0 which were mounted on ceramic structures called hybrids.

The central tracking detector was a cylindrical wire drift chamber designed to measure the direction and momentum of the charged particles as well as their energy loss, $\mathrm{dE} / \mathrm{dx}$, which provides information for particle identification. It had an overall length of $240 \mathrm{~cm}$ and an outer radius of $85 \mathrm{~cm}$, while the active volume had a length of $203 \mathrm{~cm}$ with inner and outer radii of $18.2 \mathrm{~cm}$ and $79.4 \mathrm{~cm}$, respectively. Longitudinally it covered the region from $\mathrm{Z}=-100 \mathrm{~cm}$ to $\mathrm{Z}$ $=103 \mathrm{~cm}$ resulting in a polar angular coverage of $15^{\circ}<\theta<164^{\circ}$. The resolution on the transverse momentum, $\mathrm{p}_{\mathrm{T}}$, of tracks fitted to the interaction vertex and passing at least three CTD super-layers (with $\mathrm{p}_{\mathrm{T}}>1.5 \mathrm{GeV}$ ) is :

$$
\frac{\sigma\left(p_{\mathrm{T}}\right)}{p_{\mathrm{T}}}=0.0058 p_{\mathrm{T}} \oplus 0.0065 \oplus \frac{0.0014}{p_{\mathrm{T}}},
$$

where $\mathrm{p}_{\mathrm{T}}$ is expressed in $\mathrm{GeV}$.

The three levels of the ZEUS trigger system were organized such that at each level the rate was reduced giving increased time to perform more detailed calculations. 
The high precision measurement of the integrated luminosity is essential for the determination of cross sections. At ZEUS the luminosity was determined by measuring the Bethe-Heitler QED bremsstrahlung process ep $\rightarrow e p \gamma$, where the leptons and photons are scattered at very small angles. The cross section of this process is large $\left(\sigma_{\mathrm{BH}}>20 \mathrm{mb}\right)$ and can be calculated as a function of the photon energy to an accuracy of $0.5 \%$. The precise measurement of bremsstrahlung process and its high rate allowed an accurate determination of the luminosity.

\subsubsection{Kinematics of Lepton-Proton Scattering}

In the SM, the fundamental lowest order process in lepton-proton scattering is mediated by the electroweak force through the exchange of a vector boson. If the exchanged boson is a photon, $\gamma$, or a Z, the process is called neutral current scattering (NC); in case the boson is a $\mathrm{W}^{ \pm}$, the interaction is called charged current scattering (CC). In CC scattering the final state lepton is a neutrino

$$
e^{-}+p \rightarrow v+X \text {. }
$$

where $X$ denotes the hadronic final state. The generic Feynman diagrams of both processes including the particle's momenta are shown in Figure 2. The kinematics of $e p$ collisions can be explained with the help of these diagrams as follows.

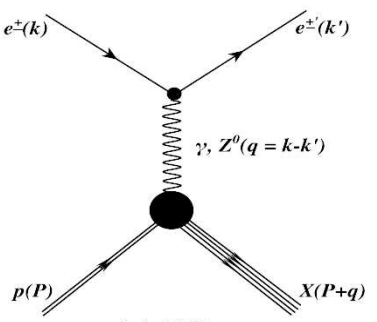

(a) $\mathrm{NC}$

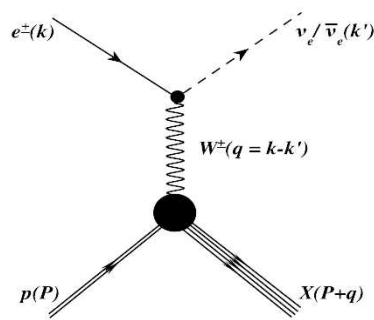

(b) $\mathrm{CC}$

Fig. 2. Electron-proton scattering: (a) in neutral current (NC), and (b) charged current (CC). The four vectors of the particles are given in parentheses.

The incoming lepton, $e^{ \pm}$, having a four-momentum $\mathrm{k}\left(\mathrm{E}_{\mathrm{e}}, \mathrm{k}\right)$ interacts with an incoming proton of four-momentum $\mathrm{P}\left(\mathrm{E}_{\mathrm{p}}, \mathrm{P}\right)$. The four-momentum of the resulting scattered lepton is $\mathrm{k}^{\prime}\left(\mathrm{E}_{\mathrm{e}}{ }^{\prime}, \mathrm{k}^{\prime}\right)$ and that of the exchanged boson is $\mathrm{q}\left(\mathrm{E}_{\gamma}, \mathrm{q}\right)$, where

$$
\mathrm{q}=\mathrm{k}-\mathrm{k} \text {. }
$$

The scattering is described by the following Lorentz-invariant variables:

$$
\begin{aligned}
s & =(k+P)^{2}, \\
Q^{2} & =-q^{2}=-\left(k-k^{\prime}\right)^{2}, 0 \leq Q^{2} \leq s, \\
y & =\frac{P \cdot q}{P \cdot k}, 0 \leq y \leq 1, \\
x & =\frac{-q^{2}}{2 P \cdot q}, 0 \leq x \leq 1,
\end{aligned}
$$

where $s$ is the total centre-of-mass energy squared. $Q^{2}$ is the negative square of the fourmomentum transfer of the exchanged boson which defines its virtuality. In the proton rest frame the inelasticity, $y$, describes the relative energy transfer from the lepton to the hadronic system and $x$ is called Bjorken scaling variable. In the quark parton model $x$ can be interpreted as the fraction of the proton momentum carried by the struck quark. 
The variable $Q^{2}$ is used to split the kinematic plane into two separate regimes, the deep inelastic scattering (DIS) and the photoproduction $(\mathrm{PhP})$ regime. Events with high photon virtuality, $Q^{2}>1 \mathrm{GeV}^{2}$, and large hadronic centre-of-mass energy, $W$, are referred to as DIS events. For these events the incoming lepton is deflected by some measurable angle and can be identified in the detector. The events with very low photon virtuality $Q^{2} \approx 0$ are characterized by the exchange of a quasi-real photon and are known as PhP events. In these events the incoming lepton is deflected at a very small angle and cannot be observed in the main detector. In DIS events the hard scale is provided by $Q^{2}$ and thus allows the perturbative calculations to be performed. For PhP events $Q^{2}$ is not a hard scale; however the transverse momentum of jets or the mass of the heavy quarks produced may define a hard scale in the event.

The virtuality, $Q^{2}$, gives the scale of the interaction. It can be thought as the "spatial" resolution of HERA to resolve the inner structure of the proton. Quantitatively it is related to the quantum wavelength, $\lambda$, of the boson which is looking into the proton, via $\lambda=h / \sqrt{ } Q^{2}$. Since the photon exchange cross section falls rapidly as a function of $Q^{2}$, at low $Q^{2}$ the photon exchange dominates the cross section over the weak bosons. Only when $Q^{2}$ is sufficiently large, are the

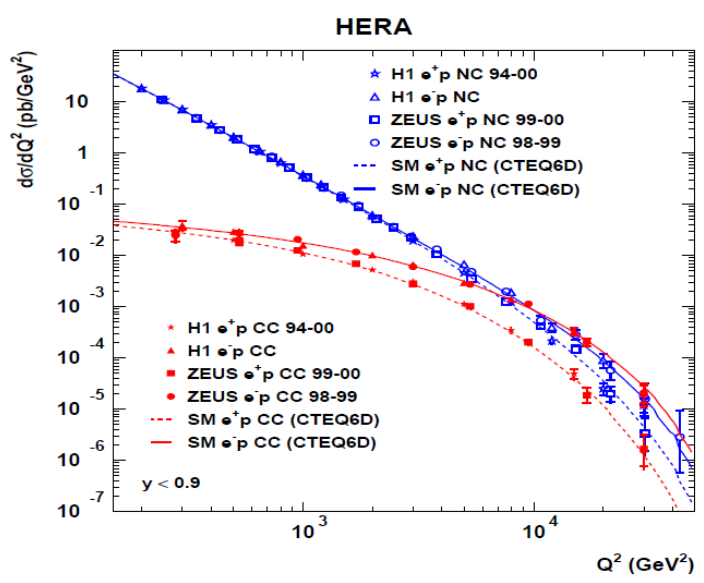
contributions from the $Z$ and $W^{ \pm}$bosons significant. Indeed, when $Q^{2} \geq M_{Z, W}^{2}$, the neutral and charged current cross sections are found to be of comparable size. The convergence of the $\mathrm{NC}$ and $\mathrm{CC}$ cross sections as seen in Figure 3 is an illustration of unification in the electroweak theory.

Fig. 3. Inclusive differential NC and CC cross sections as a function of $Q^{2}$. Data points are measurements from the ZEUS and $\mathrm{H} 1$ experiments, the lines show the SM predictions for the specific process.

\subsection{The proton under the HERA microscope}

\subsubsection{Heavy flavours}

Normal matter is mainly made of up- and down-flavoured quarks, with some admixture of virtual strange quarks. These are collectively called light flavours. In high-energy interactions, such as those that occur in electron-proton collisions at the HERA collider, the production of heavy flavours, i.e. with associated quark masses larger than the proton mass, starts to play an important role. At HERA energies, these flavours include in particular the charm quarks and the even heavier beauty quarks. In order to study the heavy flavour contributions, events containing such heavy quarks in the final state need to be tagged and separated from other processes. This can be achieved in essentially three ways. A charmed hadron (a final state particle containing a charm quark) is reconstructed by explicitly identifying and combining all its decay products. The longer lifetime or the large mass of the parent particles of such heavy flavour hadrons (charm or beauty) with respect to other particles is used to detect secondary vertices. The three methods can also be combined. At energy scales significantly larger than the proton mass, processes with quarks carrying heavy flavour quantum numbers contribute significantly to inclusive quantities like the proton structure function $F_{2}$. In the case of charm, this contribution can reach up to $36 \%$ in reactions with the highest energy transfer. In contrast to the abundant charm final states, beauty quarks appear only in a small fraction of inclusive final states at 
HERA, of the order of $1 \%$. On the other hand, the large mass and longer lifetime of the beauty hadrons makes their inclusive detection somewhat easier.

\section{ZEUS}
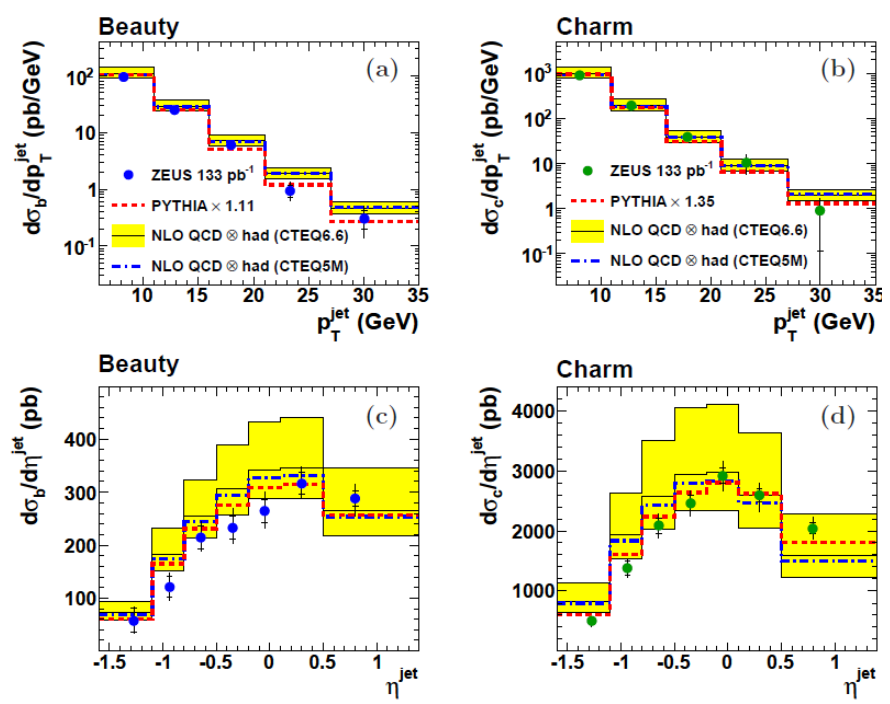

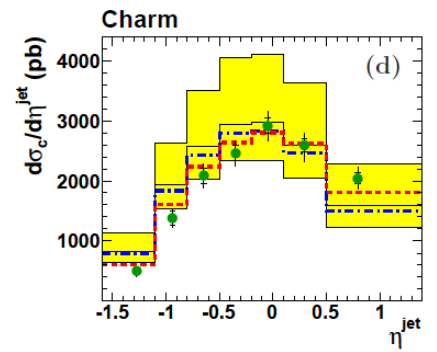

Fig. 4. Differential beauty-jet and charm-jet photoproduction cross sections as a function of (a)-(b) $p_{T}^{\text {jet }}$ and (c)-(d) $\eta^{\text {jet }}$. The data are shown as points. The inner error bars are the statistical uncertainties, while the outer error bars show the statistical and systematic uncertainties added in quadrature. The band represents the NLO QCD prediction, corrected for hadronization effects, using CTEQ6.6 as proton PDF; the shaded band shows the estimated uncertainty.

Photoproduction of beauty and charm quarks in events with at least two jets has been measured [1] with the ZEUS detector at HERA using an integrated luminosity of $133 \mathrm{pb}^{-1}$. The beauty and charm cross sections as a function of $p_{T}^{\text {jet }}$ and $\eta^{\text {jet }}$ are shown in Fig. 4. The NLO QCD predictions are in good agreement with the data and the scaled Pythia MC describes the distributions well. In Fig. 5 (left), the b-quark differential cross sections as a function of the quark transverse momentum, $d \sigma(e p \rightarrow b X) / d p^{b}{ }_{T}$, are shown for $b$-quark pseudorapidity in the laboratory frame, $\left|\eta^{b}\right|<2$, for $Q^{2}<1 \mathrm{GeV}^{2}$ and $0.2<y<0.8$. The measurement presented here extends the kinematic region to higher $p_{T}^{b}$ values than previous measurements and represents the most precise measurement of $b$-quark photoproduction at HERA
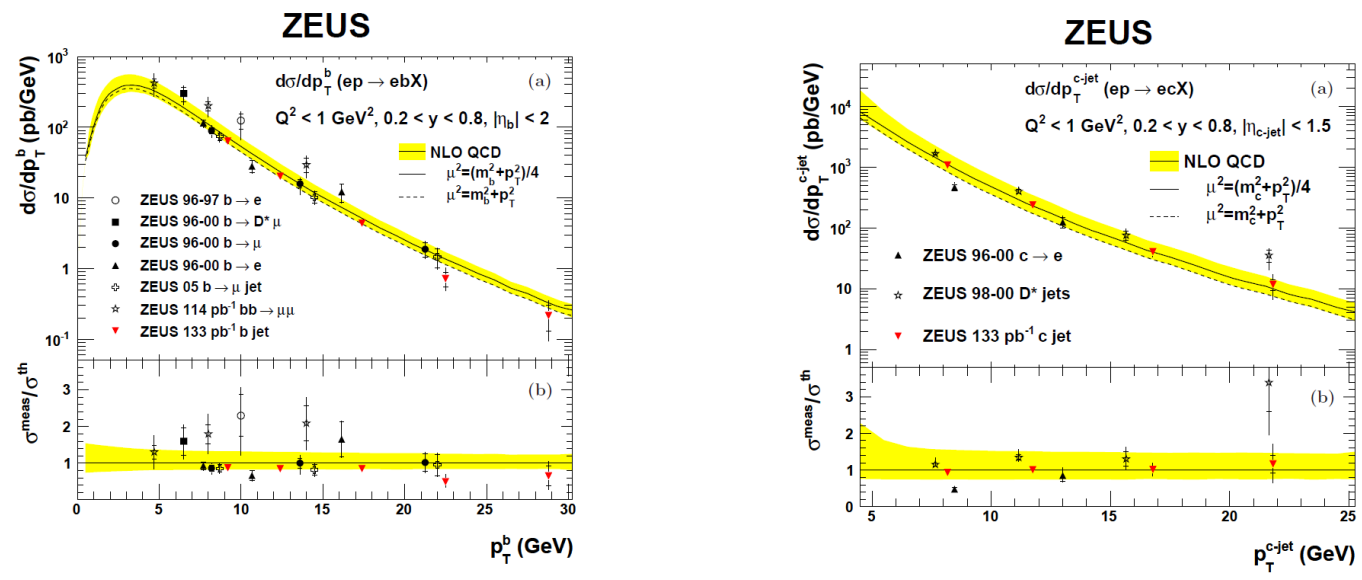

Fig. 5. Summary of differential cross sections for $b$ (left) and $c$-quark (right) production as a function of as $p_{T}^{b}$ measured by the ZEUS collaboration. The measurements are shown as points, with the results of this analysis shown as inverted triangles. The inner error bars are the statistical uncertainties, while the outer error bars show the statistical and systematic uncertainties added in quadrature. The band represents the NLO QCD prediction and its theoretical uncertainty. 
Good agreement with the NLO QCD prediction is observed for many independent ZEUS measurements, giving a consistent picture of $b$-quark photoproduction over a wide range of $p_{T}^{b}$. The corresponding $c$-quark cross sections were also calculated and are shown in Fig. 5 (right).

The production of beauty quarks in $e p$ interactions has been studied [2] with the ZEUS detector at HERA for exchanged four-momentum squared $\mathrm{Q}^{2}>10 \mathrm{GeV}^{2}$, using an integrated luminosity of $363 \mathrm{pb}^{-1}$. The beauty events were identified using electrons from semileptonic $b$ decays with a transverse momentum $0.9<p_{T}^{e}<8 \mathrm{GeV}$ and pseudorapidity $\left|\eta^{e}\right|<1.5$. Cross sections for beauty production were measured and compared with next-to-leading-order QCD calculations. The visible cross section for electrons from direct and indirect $b$-quark decays was found to be

$$
\left.\left.\sigma_{b \rightarrow e}=(71.8 \pm 5.5 \text { (stat. })_{-5.5}^{+5.3} \text { (syst. }\right)\right) \mathrm{pb}
$$

This cross section includes all electrons and positrons from both $b$ and anti- $b$ and no jet requirement was applied at the true level. This result can be compared to the HVQDIS NLO QCD prediction of

$$
\sigma_{b \rightarrow e}^{\mathrm{NLO}}=\left(67_{-11}^{+10}\right) \mathrm{pb}
$$

Differential cross sections as a function of $p_{T}^{e}$ and $\eta^{e}, Q^{2}$ and $x$ are shown in Fig. 6.

The structure function $F^{b b}$ can be defined in terms of the inclusive double-differential cross section as a function of $x$ and $Q^{2}$.

$$
\frac{\mathrm{d}^{2} \sigma_{b \bar{b}}}{\mathrm{~d} x \mathrm{~d} Q^{2}}=\frac{Y_{+}\left(2 \pi \alpha_{\mathrm{em}}^{2}\right)}{x Q^{4}}\left[F_{2}^{b \bar{b}}\left(x, Q^{2}\right)-\frac{y^{2}}{Y_{+}} F_{L}^{b \bar{b}}\left(x, Q^{2}\right)\right]
$$

where $Y+=1+(1-y)^{2}$ and $F_{L}^{b b}$ is the beauty contribution to the structure function $F_{L}$.

\section{ZEUS}
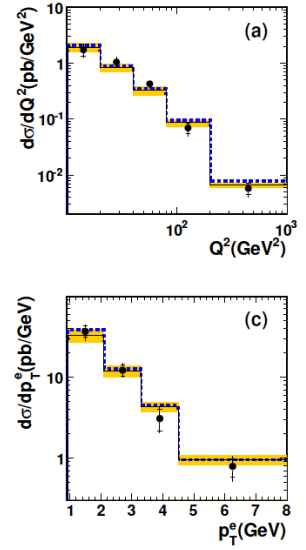
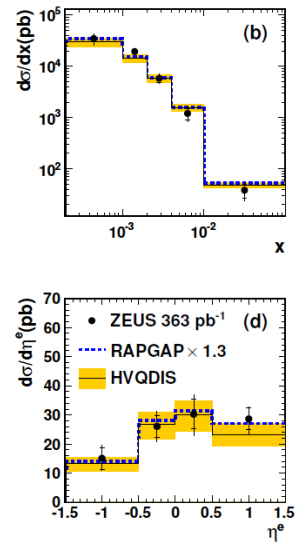

Fig. 6. Differential cross sections for electrons from bquark decays as a function of the kinematic variables (a) $Q^{2}$ and (b) $x$, and the decay electron variables (c) $p_{T}^{e}$ and (d) $\eta^{e}$.

The electron cross section, $\sigma_{b \rightarrow e}$, measured in bins of $\mathrm{x}$ and $Q^{2}$, was used to extract $F_{2}^{b b}$ at a reference point in the $\mathrm{x}-\mathrm{Q}^{2}$ plane using

$$
F_{2}^{b \bar{b}}\left(x, Q^{2}\right)=\frac{\mathrm{d}^{2} \sigma_{b \rightarrow e}}{\mathrm{~d} x \mathrm{~d} Q^{2}} \cdot \frac{F_{2}^{b b, N L U}\left(x, Q^{2}\right)}{\mathrm{d}^{2} \sigma_{b \rightarrow e}^{\mathrm{NLO}} / \mathrm{d} x \mathrm{~d} Q^{2}}
$$

The structure function $F^{b b}{ }_{2}$ is shown in Fig. 7 as a function of $x$ for nine different values of $\mathrm{Q}^{2}$ and as a function of $Q^{2}$ for fixed values of $x$. As we can see the measurement is in agreement with the results obtained from previous analyses using different techniques. For $Q^{2}>10 \mathrm{GeV}^{2}$, this measurement represents the most precise determination of $F^{b b}{ }_{2}$ by the ZEUS Collaboration. The NLO and NNLO QCD calculations provide a good description of the data.

Charm production in deep inelastic scattering has been measured [3] with the ZEUS detector at HERA using an integrated luminosity of $120 \mathrm{pb}^{-1}$. The hadronic decay channels $D^{+} \rightarrow K_{S}^{0} \pi^{+}, \Lambda_{c}^{+} \rightarrow p K_{S}^{0}$, and $\Lambda_{c}^{+} \rightarrow \Lambda \pi^{+}$, and their charge conjugates, were reconstructed. The kinematic range is $0<p_{T}\left(D^{+}, \Lambda_{c}^{+}\right)<10 \mathrm{GeV},\left|\eta\left(D^{+}, \Lambda_{c}^{+}\right)\right|<1.6,1.5<Q^{2}<1000 \mathrm{GeV}^{2}$ and $0.02<$ $y<0.7$. Inclusive and differential cross sections for the production of $\mathrm{D}^{+}$mesons are shown in Fig. 8. and compared to next-to-leading-order QCD predictions. 
ZEUS

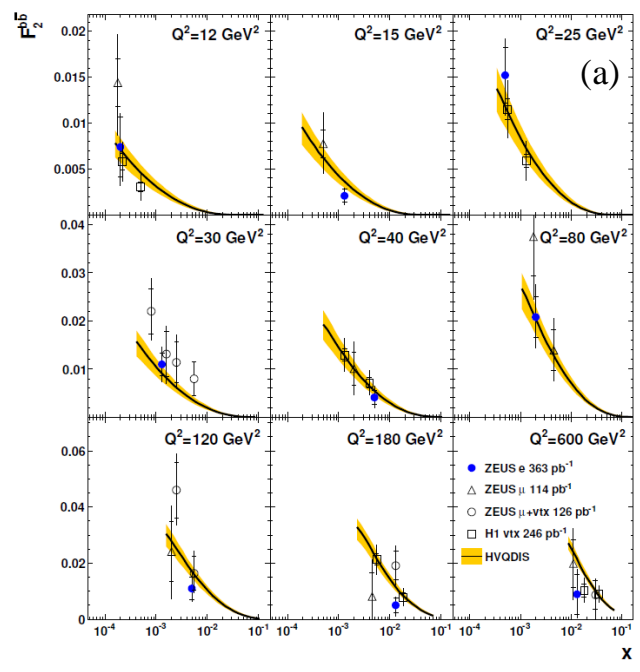

ZEUS

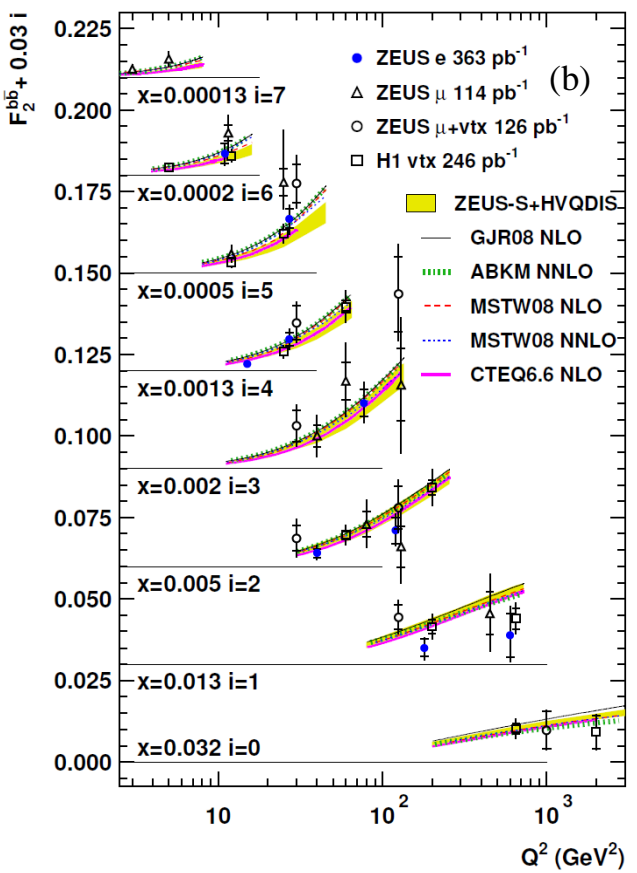

Fig. 7. The structure function $F^{b b}{ }_{2}$ (a) as a function of $x$ for nine different values of $\mathrm{Q}^{2}$; (b) as a function of $Q^{2}$ for fixed values of $x$.

The following total visible cross section for $\mathrm{D}+$ mesons was measured:

$$
\sigma\left(D^{+}\right)=25.7 \pm 4.1 \text { (stat.) }{ }_{-5.2}^{+3.8} \text { (syst.) } \pm 0.8 \text { (br.) nb. }
$$

The corresponding prediction from HVQDIS is $\sigma\left(D^{+}\right)=12.7+3.8-4.1 \mathrm{nb}$. The following $\Lambda_{c}^{+}$ cross sections were measured: using the decay channel $\Lambda_{c}{ }^{+} \rightarrow p K_{S}^{0}$

$$
\sigma\left(\Lambda_{c}^{+}\right)=14.9 \pm 4.9 \text { (stat.) }{ }_{-2.6}^{+2.2} \text { (syst.) } \pm 3.9 \text { (br.) nb; }
$$

and using the decay channel $\Lambda_{c}^{+} \rightarrow \Lambda \pi^{+}$

$$
\sigma\left(\Lambda_{c}^{+}\right)=14.0 \pm 5.8 \text { (stat.) }{ }_{-3.3}^{+3.8} \text { (syst.) } \pm 3.7 \text { (br.) nb }
$$

The cross sections obtained using

ZEUS
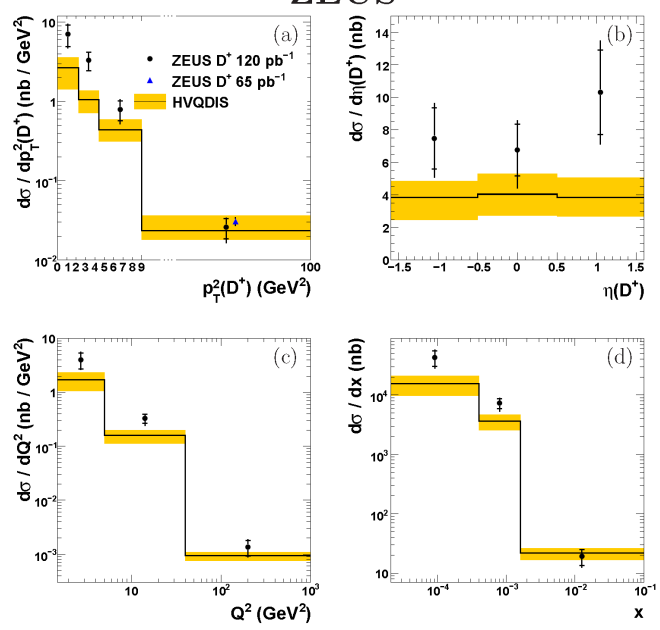

different decay channels are in good agreement.

Figure 8: Differential $\mathrm{D}^{+}$cross sections as a function of (a) $p^{2}{ }_{T}\left(D^{+}\right),\left(\right.$b) $\eta\left(D^{+}\right)$, (c) $Q^{2}$ and (d) $x$ compared to the NLO QCD calculation of HVQDIS. The measured cross sections are shown as dots and the triangle represents a previous ZEUS result. 


\subsubsection{Proton structure}

The collisions of electrons or positrons with protons at HERA allow the measurement of the proton content (quark and gluons) with unprecedented precision. Most of the electron-proton interactions are taking place at a low momentum transfer $Q^{2}$ and are mediated by a virtual photon. A small but significant part of the collisions correspond to the very high momentum transfers, from a thousand up to a few tens of thousands of $\mathrm{GeV}^{2}$. In this domain, which was reached in deep-inelastic scattering for the first time at HERA, the collisions are also mediated by weak bosons, leading to events with spectacular signatures. In the case of at high $Q^{2}$, the incoming electron is scattered at wide angles, similar to the classical Rutherford experiment, thereby probing the point-like nature of the struck quarks with resolutions down to $10^{-18} \mathrm{~m}$. For this type of interactions, contributions from the neutral weak $Z^{0}$ boson are expected. The interactions at high $Q^{2}$ can also be mediated by charged weak bosons $W^{ \pm}$, leading to final states with significant imbalance in the detected transverse momentum, due to the elusive neutrino produced as a result of the electron conversion. These charged-current interactions can be measured at HERA. The DIS processes at large momentum transfer offer new possibilities to pin down the proton structure functions. Previous results for this region where obtained using fixed nuclear targets, in a regime where the momentum transfer barely reached a few tens of $\mathrm{GeV}^{2}$. At HERA, the absence of nuclear media around the proton allows a clean determination of the parton distribution functions from the measured structure functions. The PDFs are defined as the probability densities of given partons (quarks of different flavours and gluons) to carry a fraction $\mathrm{x}$ of the proton momentum.

The measurements based on the data collected by H1 and ZEUS during the first phase from 1994 to 2000 (HERA I run) were used to accurately determine the proton structure. An unprecedented precision of about 1-2 \% was obtained at low $Q^{2}$, while the accuracy of the high $Q^{2}$ measurements was still dominated by statistical precision. The HERA II data sets were recorded after a luminosity upgrade and various detector improvements by $\mathrm{H} 1$ and ZEUS in the period from 2003 and 2007. They were analyzed by both collaborations and combined during the year 2010. Finally they were also combined with the HERA I data. The gain in statistics is supplemented by an appropriate treatment of the systematic uncertainties, leading to a significant improvement in precision.

The combined cross section measurements from HERA I and HERA II are used to obtain the parton distribution function set called HERAPDF 1.5. The PDFs and their uncertainties obtained are shown in Fig. 9. The fit was performed with 14 free PDF parameters. As was the case for the previous fits based on HERA data only, the HERAPDF 1.5 set has the advantage to be based on a coherent data set from one collider only, composed from precise and

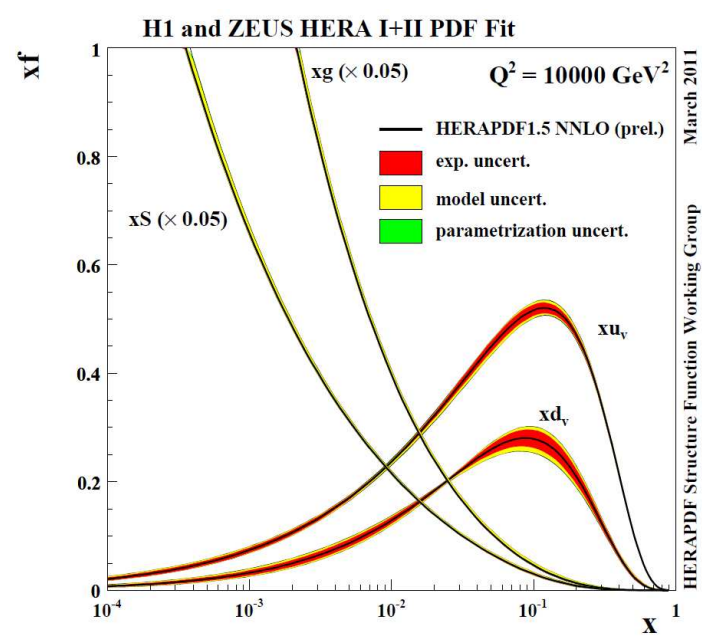
mutually consistent samples produced by two independent experiments. The universality of the PDFs assumes that other processes and collision configurations involving protons can be calculated using the PDFs extracted from precision measurements. In particular, HERAPDF1.5 sets can be used to predict cross sections for phenomena occurring in proton-proton collisions at the LHC.

Fig. 9. The improved precision of the parton distribution functions obtained by using combined HERA II data shown at a scale $Q^{2}=10000 \mathrm{GeV}^{2}$, typical for the expected interactions at the LHC. 
The HERA inclusive data analysis is now close to its ultimate precision. Only the combination of the inclusive data with further semi-inclusive measurements (charm or jets) may allow a further increase in precision in various regions of the phase space and provide a precise test of the theory of strong interactions in DIS.

\subsubsection{Strong interactions}

Strong interactions result from the exchange of messenger particles, gluons, between the matter constituents of nucleons and nuclei, the quarks. The data from the electron-proton collider HERA are very well suited to study the properties of the underlying theory, quantum chromodynamics. Insights obtained at the "HERAscale" of about 10-100 GeV can be successfully applied to the "Terascale" probed by the LHC. Using the knowledge of this internal structure, based to a large extent on measurements at HERA, one can go a step further and measure effects which are solely due to QCD. One such effect is the probability of a quark to absorb or radiate a gluon. This probability is parameterized in terms of the strong coupling constant, $\alpha_{s}$. It has been well known since decades that its value rather depends on the energy scale of the interaction and decreases with increasing energy or decreasing distance scale (asymptotic freedom). On the other hand, the coupling increases with increasing distance scale, such that at some point the probability for further interactions reaches the order of unity. Thus a quark or gluon resulting from a high-energy process will always "fragment" into a whole bundle of secondary particles, called a jet, when it moves away from the interaction.

Measurements of jet cross sections are a well-established tool for QCD studies and have been performed for many different observables at HERA. For jet analyses in neutral current deep inelastic scattering, the Breit reference frame is preferred, since it provides a maximal separation between the products of the beam fragmentation and the hard jets. In this frame, the exchanged space-like virtual boson has 3-momentum $q=(0,0,-Q)$ and is collinear with the incoming parton. While retaining hard QCD processes at leading order in the strong coupling constant $\alpha_{s}$, the contribution from the parton-model process can be suppressed by requiring the production of jets with high transverse energy in the Breit frame. Therefore, measurements of
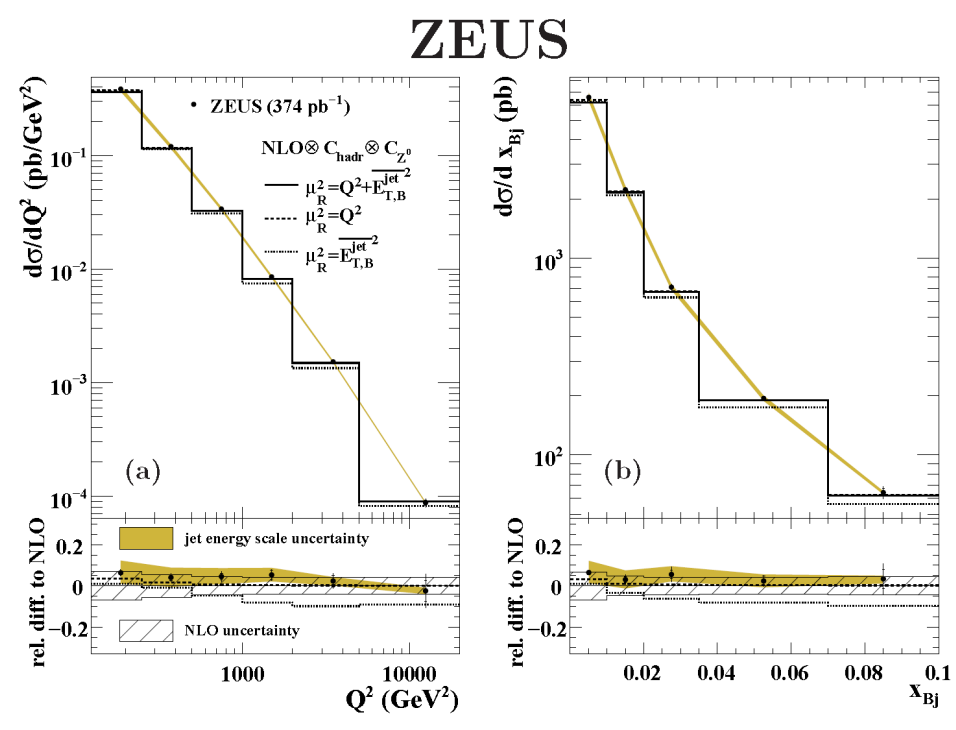
jet cross sections in the Breit frame are directly sensitive to hard QCD processes, allowing tests of perturbative QCD (pQCD), of the factorisation ansatz and of the parton distribution functions of the proton.

Fig. 10. The measured differential cross-sections (a) $d \sigma / d Q^{2}$ and (b) $d \sigma / d x_{B j}$ for inclusive dijet production 
Single- and double-differential inclusive dijet cross sections in neutral current deep inelastic ep scattering have been measured [4] with the ZEUS detector using an integrated luminosity of $374 \mathrm{pb}^{-1}$. The measurement was performed at large values of the photon virtuality, $Q^{2}$, between 125 and $20000 \mathrm{GeV}^{2}$.

The cross sections are described by the predictions of next-to-leading-order QCD. The single-differential dijet cross-sections $d \sigma / d Q^{2}$ and $d \sigma / d x_{B j}$ are shown in Fig.10 (a) and (b). Here, $x_{B j}$ is the Bjorken scaling variable that defines, for the parton-model process, the fraction of the proton momentum carried by the struck massless parton.

At large boson virtuality, $Q^{2}$, the experimental and theoretical systematic uncertainties are small and, thus, use of the large HERA data sample can provide powerful physical constraints.

The probability to radiate one or two extra "hard" gluons in the final state is theoretically calculable. Such gluons will influence the shape of the energy flow in the final state, and therefore the details of how jets are reconstructed from this energy flow. Different jet algorithms exist which are sensitive to these details in different ways. Fig. 11 shows the dependence of $\alpha_{s}$

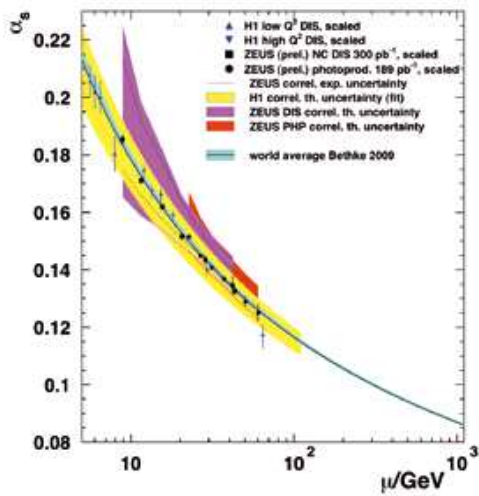
on the energy scale, as extracted from several HERA data sets of this kind. The decrease towards higher energy scales is clearly visible, and again well described by QCD calculations. The expected continuation of this dependence into the Terascale regime can be very reliably predicted.

Fig. 11. The running coupling constant $\alpha_{s}$ as a function of the energy scale $\mu$.

Inclusive-jet cross sections were measured [5] in the reaction $e p \rightarrow e+j e t+X$ for photon virtuality $Q^{2}<1 \mathrm{GeV}^{2}$ and $\gamma p$ centre-of-mass energies in the region $142<W_{\gamma p}<293 \mathrm{GeV}$ with the ZEUS detector at HERA using an integrated luminosity of $300 \mathrm{pb}^{-1}$. Jets were identified using the $\mathrm{kT}$, anti-kT or SIScone jet algorithms in the laboratory frame. Single-differential cross sections are measured as functions of the jet transverse energy, $E^{j e t}{ }_{T}$, and pseudorapidity, $\eta^{\text {jet }}$, for jets with $E^{j e t}{ }_{T}>17 \mathrm{GeV}$ and $-1<\eta^{j e t}<2.5$ and are presented in Fig. 12. The NLO QCD predictions are compared to the measurements in these figures. The calculation reproduces the measured $d \sigma / E_{T}^{j e t}$ well, except at the lowest $E_{T}^{\text {jet }}$ bin. No significant deviation is observed in the highest $E_{T}^{\text {jet }}{ }_{T}$ region. In addition, measurements of double-differential inclusive-jet cross sections are measured as functions of $E^{j e t}{ }_{T}$ in different regions of $\eta^{\text {jet}}$, which have the potential to constrain the gluon density in the proton and the photon. Next-to-leading-order QCD calculations give a good description of the measurements. The measured single-differential cross-sections based on the three jet algorithms were used to determine values of $\alpha_{s}\left(M_{Z}\right)$. The NLO QCD calculations were performed with five sets of the ZEUS-S proton PDFs which were determined from global fits assuming different values of $\alpha_{s}\left(M_{Z}\right)$. The GRV-HO set was used as default for the photon PDFs. 


\section{ZEUS}

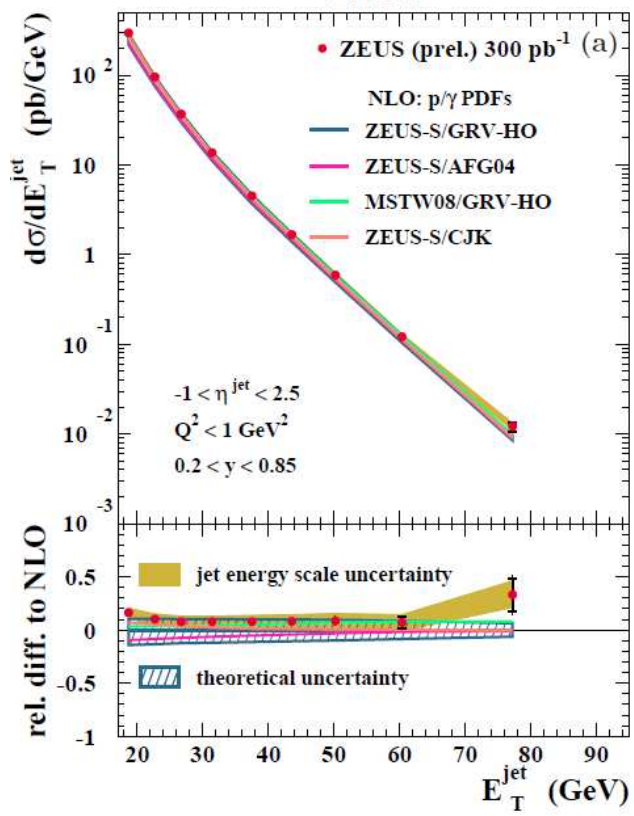

ZEUS

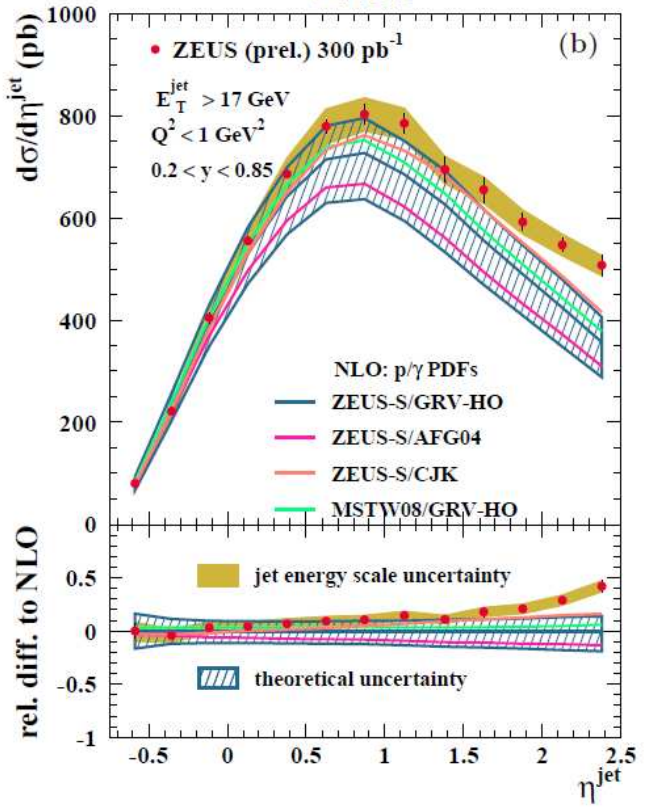

Fig.12. The measured differential cross-section $d \sigma / E_{T}^{\text {jet }}$ (a) and $d \sigma / \eta^{\text {jet }}$ (b) for inclusive-jet photoproduction with $E_{T}^{\text {jet }}>17 \mathrm{GeV}$ and $-1<\eta^{\text {jet }}<2.5$ (dots) in the kinematic range given by $\mathrm{Q}^{2}<1$ $\mathrm{GeV}^{2}$ and $0.2<\mathrm{y}<0.85$. For comparison, the NLO QCD calculation with $\mu=E_{T}^{j e t}$ are shown.

The value of $\alpha_{s}\left(M_{Z}\right)$. used in each calculation was that associated with the corresponding set of proton PDFs.

$$
\begin{aligned}
\left.\alpha_{s}\left(M_{Z}\right)\right|_{k_{T}} & =0.1206_{-0.0022}^{+0.0023} \text { (exp.) }{ }_{-0.0033}^{+0.0042} \text { (th.), } \\
\left.\alpha_{s}\left(M_{Z}\right)\right|_{\text {anti- } k_{T}} & =0.1198_{-0.0022}^{+0.0023} \text { (exp.) }{ }_{-0.0032}^{+0.0041} \text { (th.) and } \\
\left.\alpha_{s}\left(M_{Z}\right)\right|_{\text {SIScone }} & =0.1196_{-0.0021}^{+0.0022} \text { (exp.) }{ }_{-0.0042}^{+0.0046} \text { (th.). }
\end{aligned}
$$

The values of $\alpha_{s}\left(M_{Z}\right)$ determined from the anti-kT (SIScone) measurements are consistent with that obtained from the kT analysis within $-0.7(-0.8) \%$.

\section{ZEUS}

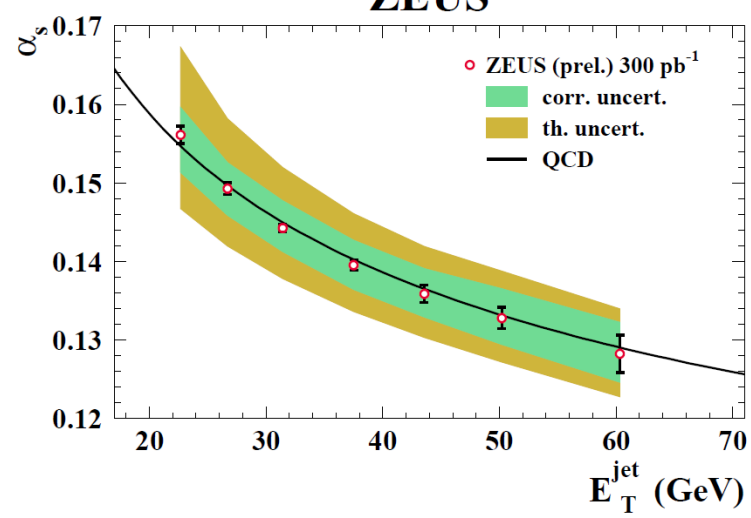

Fig. 13. The $\alpha_{s}$ values determined from the measured $d \sigma / E^{\text {jet }}{ }_{T}$ as a function of $E^{\text {jet }}{ }_{T}$ (open circles). The error bars represent the uncorrelated uncertainties of the data; the inner shaded area represents the correlated systematic uncertainties and the outer shaded area represents the correlated and theoretical uncertainties added in quadrature. The solid line indicates the renormalisation-group prediction at two loops obtained from the $\alpha_{s}\left(M_{Z}\right)$ value determined in this analysis. 
The energy-scale dependence of $\alpha_{s}$ was determined from a NLO QCD fit to the measured $d \sigma / E_{T}^{j e t}$ cross section based on the kT jet algorithm. Values of $\alpha_{s}$ were extracted at each mean value of measured $E^{\text {jet }}{ }_{T}$ without assuming the running of $\alpha_{s}$. The extracted values of $\alpha_{s}$ as a function of $E_{T}^{j e t}$ are shown in Fig.13. The results are in good agreement with the predicted

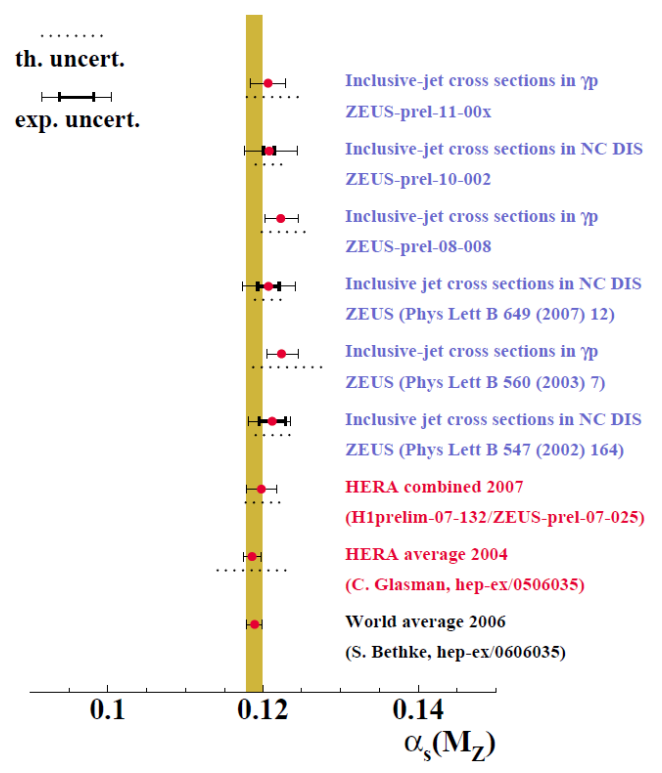

running of the strong coupling calculated at two loops over a large range in $E^{j e t}$ from a single experiment with high experimental precision. Fig. 14 shows the result of this analysis compared to previous ZEUS analyses.

Fig. 14. Extracted $\alpha_{s}\left(M_{Z}\right)$ value from this analysis (upper dot). For comparison, other determinations from ZEUS, the HERA combined 2007, the HERA average 2004 and the world average 2006 values are also shown. The shaded band represents the uncertainty of the world average.

The production of isolated high-energy photons accompanied by jets has been measured in deep inelastic ep scattering with the ZEUS detector [6] at HERA using an integrated luminosity of $320 \mathrm{pb}^{-1}$. Events in which an isolated high-energy photon is observed provide a direct probe of underlying partonic process in high-energy collisions involving photons, since the emission of such photons is unaffected by parton hadronization. Measurements were made for exchanged photon virtuality, $Q^{2}$, in the range 10 to $350 \mathrm{GeV}^{2}$. The photons were measured in the transverse and pseudorapidity ranges $4<E_{T}^{\gamma}<15 \mathrm{GeV}$ and $-0.7<\eta^{\gamma}<0.9$, and the jets were measured in the transverse energy and pseudorapidity ranges $2.5<E_{T}^{\text {jet }}<35 \mathrm{GeV}$ and $-1.5<\eta^{\text {jet }}<1.8$. Differential cross sections are presented as functions of these quantities. This measurement provides a more detailed test of perturbative QCD in a kinematic region with two hard scales, given by $Q^{2}$ and $E_{T}^{\gamma}$, the transverse energy of the emitted photon or, equivalently, the momentum transfer in the QCD scatter. The differential cross sections as functions of $E_{T}^{\gamma}, \eta^{\gamma}$, $Q^{2}$ and $x$ are shown in Fig. 15. Leading-logarithm parton-shower Monte-Carlo and perturbative QCD predictions are compared to the measurements. Perturbative QCD predictions give a reasonable description of the data over most of kinematic range. There are two contributions to the scattering cross section for the $e p \rightarrow e \gamma X$. One of these contributions comes from the radiation of a photon from the quark line (called $Q Q$ photons) and a second from the radiation from the lepton line (called $L L$ photons). 


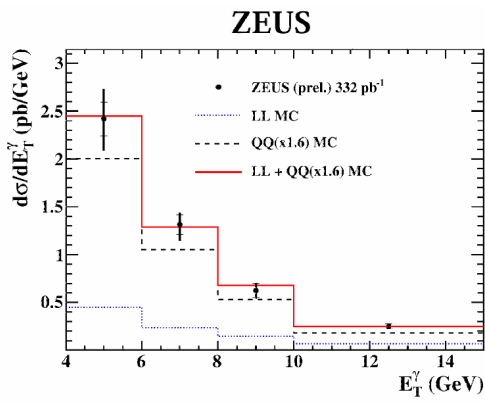

(a)

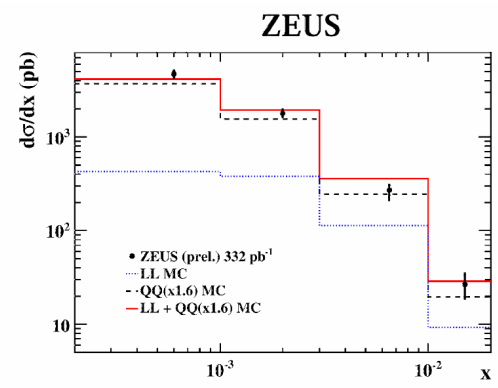

(c)

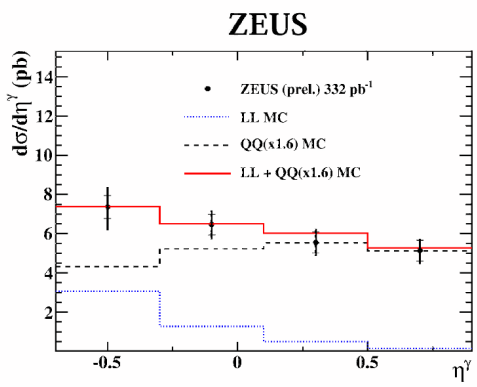

(b)

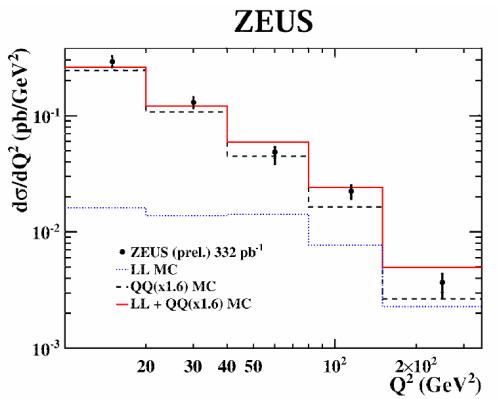

(d)

Fig. 15. Isolated photon differential cross sections in (a) $E_{T}^{\gamma}$, (b) $\eta^{\gamma}$, (c) $x$ and (d) $Q^{2}$. The inner and outer error bars show, respectively, the statistical uncertainty and the statistical and systematic uncertainties added in quadrature. The solid histograms are the Monte-Carlo predictions from the sum of QQ photons from Pythia normalized by a factor 1.6 plus Djangon LL photons. The dashed (dotted) lines show the QQ (LL) contributions.

\section{References}

[1] H. Abramowicz et al. Measurement of heavy-quark jet photoproduction at HERA, Eur.Phys.J. C71 (2011) 1659, DESY-11-067, arXiv:1104.5444 [hep-ex]

[2] H. Abramowicz et al. Measurement of beauty production in deep inelastic scattering at HERA using decays into electrons, Eur.Phys.J. C71 (2011) 1573, DESY-11-005, arXiv:1101.3692

[3] H. Abramowicz et al. Measurement of $D+$ and $\Lambda+c$ production in deep inelastic scattering at HERA. JHEP 1011 (2010) 009, DESY-10-064, arXiv:1007.1945 [hep-ex]

[4] H. Abramowicz et al. Inclusive dijet cross sections in neutral current deep inelastic scattering at HERA, Eur.Phys.J.C70:965-982,2010, DESY-10-170, arXiv:1010.6167 [hep-ex]

[5] H. Abramowicz et al. ZEUS Collaboration, Inclusive-jet cross sections in photoproduction at HERA and determination of $\alpha_{s}$, ZEUS-prel-11-005, 2011, in proceedings of XIX International Workshop on Deep-Inelastic Scattering and Related Subjects (DIS 2011)11-15 April 2011, Newport News, VA USA

[6] H. Abramowicz et al. ZEUS Collaboration, Isolated-photon+jet production in NC DIS at HERA, ZEUS-prel-11-007, 2011, in proceedings of XIX International Workshop on Deep-Inelastic Scattering and Related Subjects (DIS 2011)11-15 April 2011, Newport News, VA USA 